\title{
Rapid virtual hematoxylin and eosin histology of breast tissue specimens using a compact fluorescence nonlinear microscope
}

\author{
Lucas C Cahill ${ }^{1,2}$, Michael G Giacomelli ${ }^{1}$, Tadayuki Yoshitake ${ }^{1}$, Hilde Vardeh ${ }^{3}$, Beverly E Faulkner-Jones ${ }^{3}$, \\ James L Connolly ${ }^{3}$, Chi-Kuang Sun ${ }^{4}$ and James G Fujimoto ${ }^{1}$
}

Up to $40 \%$ of patients undergoing breast conserving surgery for breast cancer require repeat surgeries due to close to or positive margins. The lengthy processing required for evaluating surgical margins by standard paraffin-embedded histology precludes its use during surgery and therefore, technologies for rapid evaluation of surgical pathology could improve the treatment of breast cancer by reducing the number of surgeries required. We demonstrate real-time histological evaluation of breast cancer surgical specimens by staining specimens with acridine orange (AO) and sulforhodamine 101 (SR101) analogously to hematoxylin and eosin (H\&E) and then imaging the specimens with fluorescence nonlinear microscopy (NLM) using a compact femtosecond fiber laser. A video-rate computational light absorption model was used to produce realistic virtual H\&E images of tissue in real time and in three dimensions. NLM imaging could be performed to depths of $100 \mu \mathrm{m}$ below the tissue surface, which is important since many surgical specimens require subsurface evaluation due to contamination artifacts on the tissue surface from electrocautery, surgical ink, or debris from specimen handling. We validate this method by expert review of NLM images compared to formalinfixed, paraffin-embedded (FFPE) H\&E histology. Diagnostically important features such as normal terminal ductal lobular units, fibrous and adipose stromal parenchyma, inflammation, invasive carcinoma, and in situ lobular and ductal carcinoma were present in NLM images associated with pathologies identified on standard FFPE H\&E histology. We demonstrate that AO and SR101 were extracted to undetectable levels after FFPE processing and fluorescence in situ hybridization (FISH) HER2 amplification status was unaffected by the NLM imaging protocol. This method potentially enables cost-effective, real-time histological guidance of surgical resections.

Laboratory Investigation (2018) 98, 150-160; doi:10.1038/labinvest.2017.116; published online 13 November 2017

Breast cancer is the second most common form of malignancy in American women, with over 300000 cases diagnosed annually. ${ }^{1}$ The standard of care for early-stage breast cancer is breast conserving therapy (BCT) which includes surgery to remove the tumor followed by adjuvant radiation therapy and possibly chemotherapy to eradicate residual disease. ${ }^{1,2}$ Unfortunately, due to the difficulty assessing tumor margins during surgery and the desire to conserve breast tissue for aesthetic outcome, up to $40 \%$ of all women who receive BCT require repeat surgeries due to positive or close margins, ${ }^{3-8}$ resulting in increased morbidity, worsened cosmetic outcomes, delayed radiotherapy, and greatly increased healthcare costs. Formalin-fixed, paraffin-embedded hematoxylin and eosin (FFPE H\&E) histopathology is the gold standard for evaluating breast surgical margins, however, the multi-hour processing time of fixation, embedding, microtoming and staining, precludes rapid evaluation of histology. Frozen section analysis (FSA) enables rapid tissue processing and evaluation, but has reduced accuracy when compared to standard FFPE $\mathrm{H} \& \mathrm{E}$ and is particularly challenging in fatty tissue due to freezing artifacts and physical sectioning difficulties. ${ }^{9,10}$ Furthermore, both of these techniques are labor-intensive, resulting in substantial pressure from both the Centers for Medicare and Medicaid Services and private insurers to control costs. ${ }^{11}$

Department of Electrical Engineering and Computer Science and Research Laboratory of Electronics, Massachusetts Institute of Technology, Cambridge, MA, USA ${ }^{2}$ Harvard-MIT Division of Health Sciences and Technology, Massachusetts Institute of Technology, Cambridge, MA, USA; ${ }^{3}$ Department of Pathology, Beth Israel Deaconess Medical Center, Harvard Medical School, Boston, MA, USA and ${ }^{4}$ Department of Electrical Engineering and Graduate Institute of Photonics and Optoelectronics, National Taiwan University, Taipei, Taiwan

Correspondence: Dr JG Fujimoto, PhD, Department of Electrical Engineering and Computer Science and Research Laboratory of Electronics, Massachusetts Institute of Technology, 77 Massachusetts Avenue \#36-361, Cambridge, MA, USA; jgfuji@mit.edu

Received 4 May 2017; revised 7 September 2017; accepted 8 September 2017 
Therefore, new technologies for rapid and cost-effective histological evaluation of surgical pathology could improve breast cancer treatment, while reducing health care costs by reducing re-excision rates. Optical sectioning microscopy enables rapid, high-resolution imaging of freshly excised tissue specimens by imaging a selected narrow depth range within tissue without requiring physical sectioning on a microtome. ${ }^{12}$ Confocal fluorescence microscopy (CFM) ${ }^{13-17}$ structured illumination microscopy (SIM), ${ }^{18,19}$ stimulated Raman scattering ${ }^{20}$ and nonlinear microscopy $(\mathrm{NLM})^{21-23}$ have all been reported to enable optical sectioning and histological evaluation of pathology in freshly excised, unfixed tissue without microtoming. Of these methods, NLM is uniquely attractive because nonlinear fluorescent excitation enables high-resolution imaging through overlaying tissue, blood, or cellular debris into surgical specimens at extremely high imaging rates relative to other methods. Furthermore, recent studies have demonstrated a high rate of concordance with conventional paraffin-embedded histopathology for the evaluation of breast surgical specimens. ${ }^{21}$

Unfortunately, NLM typically requires femtosecond Titanium:Sapphire (Ti:S) lasers, which are large, fragile, expensive, and require regular maintenance. Alternatively, a few previous works have used high power amplified fiber laser systems, ${ }^{24,25}$ which are more compact, but still relatively costly. A more cost-effective alternative is to use lower power, unamplified femtosecond ytterbium fiber lasers that operate at $1 \mu \mathrm{m}$ wavelengths. These lasers are widely used for industrial applications because of their low cost, compact size, and excellent reliability, important requirements for an intraoperative diagnostic tool. Furthermore, a $1 \mu \mathrm{m}$ wavelength is advantageous because there is less signal attenuation than for shorter wavelengths, enabling deeper imaging into tissue $^{26-28}$ analogous to serial sectioned histology. Subsurface imaging is important in intraoperative scenarios where the specimen surfaces typically have contamination due to electrocautery, surgical ink or debris from specimen handling, making pathologies difficult to visualize.

Reproducing the established diagnostic features of H\&Estained tissue using fluorescence NLM requires nuclear specific contrast with stromal counterstaining analogous to hematoxylin and eosin. These fluorescent stains must be spectrally separable from each other to discriminate between structures, rapidly penetrate live tissue, avoid interference with subsequent histological processing or assays, and be costeffective. 4',6-diamidino-2-phenylindole (DAPI), a blue nuclear fluorophore, has been used with eosin, a strong red fluorophore, to provide H\&E-like contrast in fluorescence microscopy, ${ }^{29}$ however, two photon excitation of DAPI requires shorter wavelengths (two photon excitation maximum at $\sim 0.7 \mu \mathrm{m})$ and therefore cannot be used with inexpensive ytterbium fiber lasers. Acridine orange $(\mathrm{AO})$ is a common nuclear stain that has been used in CFM, ${ }^{30}$ SIM, ${ }^{19}$ and NLM. ${ }^{21}$ AO rapidly binds to DNA, has a broad absorption spectrum and high-quantum yield. Second harmonic generation $(\mathrm{SHG})^{21}$ and $\operatorname{eosin}^{31}$ have been combined with $\mathrm{AO}$ to provide stromal contrast, however, the former requires high incident powers and has poor specificity for eosinophilic structures and the latter overlaps emission spectra with $\mathrm{AO}$ making separation of signal complicated. Sulforhodamine 101 (SR101) is a low cost, red fluorescent fluorone stain with a structure closely related to that of eosin. SR101 stains cytosol and collagen similarly to $\operatorname{eosin}^{32}$ and rapidly penetrates through live tissue. Furthermore, the red emission makes it spectrally-separable from $\mathrm{AO}$, while the large two photon absorption at $1 \mu \mathrm{m}$ lowers laser power requirements.

In this work, we describe a technique for rapid histological evaluation of freshly excised, unfixed breast tissue specimens using fluorescence NLM with a compact, low cost fiber laser (Figure 1). A rapid staining procedure using $\mathrm{AO}$ and SR101 generates images analogous to FFPE H\&E slides at video-rate (16 frames per second). We demonstrate that the fluorescent stains are extracted to undetectable levels by standard tissue processing using a commercial histology processor (ethanol dehydration followed by xylene) and do not affect HER2 amplification status via fluorescent in situ hybridization (FISH) assay. We present a comparative study of freshly excised, unfixed breast tissue pathology using virtual H\&E nonlinear microscope images and standard FFPE H\&E.

\section{MATERIALS AND METHODS Tissue Preparation}

To demonstrate rapid imaging for real-time histological evaluation of breast tissue, breast tissue not required for diagnostic purposes was collected from patients undergoing mastectomy or lumpectomy. All research was performed according to protocols approved by Massachusetts Institute of Technology Committee on the Use of Humans as Experimental Subjects and Beth Israel Deaconess Medical Center Committee on Clinical Investigations and Institutional Review Board. The requirement for informed consent was waived by both committees because specimens were deidentified discarded human tissue, not required for clinical diagnosis.

Unfixed, inked surgical specimens were procured within hours of surgery, dissected to reveal representative pathology and stained in a solution of $160 \mu \mathrm{M}$ AO and $65 \mu \mathrm{M}$ SR101 dissolved in 1:1 water: ethanol for $2 \mathrm{~min}$, then rinsed in 1:1 water: ethanol for 20 seconds. The 1:1 water: ethanol solution for staining is necessary to prevent the crystallization of SR101 that occurs when AO and SR101 are mixed together in pure water. With a 2 min staining time, AO and SR101 penetrated up to $400 \mu \mathrm{m}$ from the surface into the tissue, although staining times as short as $30 \mathrm{~s}$ enable deep volumetric NLM imaging (Supplementary Figure S1). The stained specimens were transferred to a modified histology processing cassette with a glass coverslip on the front to provide a flat imaging surface for NLM. After NLM imaging, the specimens were fixed in $10 \%$ (vol/vol) neutral buffered formalin while 
immobilized against the coverslip to maintain tissue orientation of the imaged surface, then processed, paraffinembedded, physically sectioned, and stained with H\&E. The $H \& E$ slides were scanned and linearly scaled to match the size of the freshly excised, unfixed tissue (as recorded by optical encoders on the NLM precision microscope stage prior to fixation) to account for minor tissue deformation $(<10 \%)$ due to paraffin embedding and microtoming.

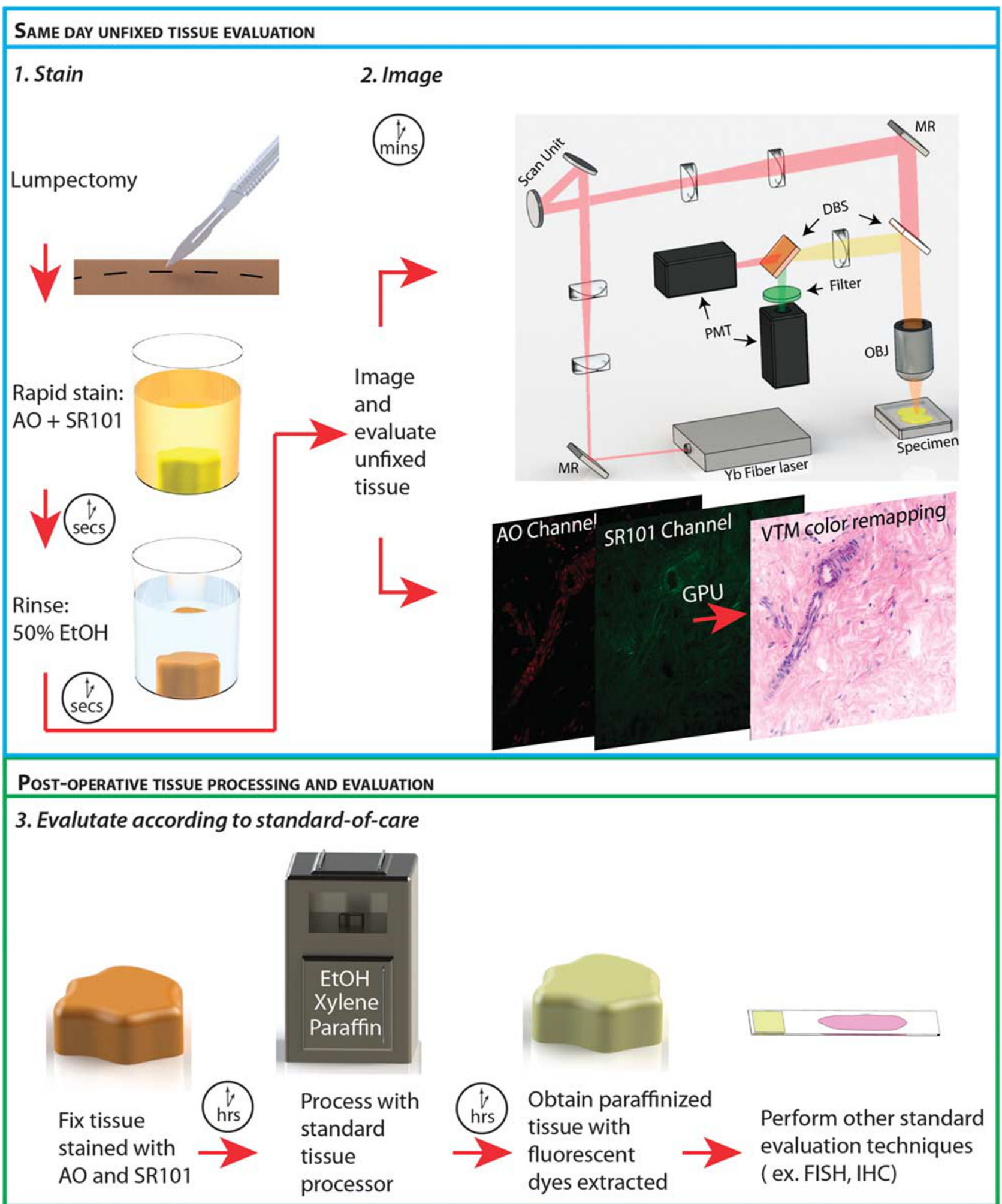




\section{Image Acquisition}

Figure 1 shows a schematic diagram of the NLM instrument. The light source was a $1.03 \mu \mathrm{m}$ wavelength ytterbium fiber laser (Origami-10, OneFive, Regensdorf, Switzerland) generating $170 \mathrm{fs}$ pulses at $100 \mathrm{MHz}$ repetition rate. The laser beam was scanned with an $8 \mathrm{kHz}$ resonant scanner $(16 \mathrm{kHz}$ bi-directional line scan rate) and a perpendicular galvanometer scanner through a $20 \times, 1.0 \mathrm{NA}$ water immersion objective (XLUMPFL20XW 1.0 NA, Olympus, Tokyo, Japan). The incident average power was $\leq 65 \mathrm{~mW}$ on the specimen and the fluorescent emission light from the AO and SR101 was collected with a dichroic beam splitter (DBS) $(590 \mathrm{~nm}$ cutoff) and directed to two photomultiplier tubes (PMT; H7422-40p, Hamamatsu, Japan). An additional $540 \pm 20 \mathrm{~nm}$ bandpass filter was used in the $\mathrm{AO}$ channel to reduce autofluorescence. $1024 \times 1024$ pixel frames with a $490 \times 490 \mu \mathrm{m}$ field of view were acquired at 16 frames per second and mosaicked by a linear motor translating stage (MLS203, Thorlabs, NJ, USA). Images at planes below the specimen surface could be acquired by adjusting the objective focus.

\section{Image Processing}

Fluorescent NLM images were rendered into an H\&E color display using a computer algorithm called virtual transillumination microscopy (VTM). ${ }^{33}$ In VTM, the concentration of dye or fluorophore is estimated based on the fluorescence intensity and converted to a transillumination absorption using the Beer-Lambert law, which relates dye concentration to absorption. This enables physically realistic rendering of epi-detected NLM images as FFPE transillumination $\mathrm{H} \& \mathrm{E}$ images in real time and at video rate ${ }^{33}$ enabling the microscope to be operated like a conventional histological microscope. However, to compare NLM images to FFPE H\&E slide images, frames from each specimen location were recorded with 10 -fold averaging at constant magnification and then stitched into mosaic images to create large field of view single plane images at a given depth below the specimen surface. This process takes $\sim 4.75 \mathrm{~s} \mathrm{~mm}^{-2}$, which is an artificially long delay and only required for comparison to FFPE H\&E sections. In a clinical setting, the instrument would be operated in real-time mode analogously to a conventional histological microscope, without requiring mosaicking.

\section{Reading and Analysis}

NLM and corresponding FFPE H\&E images were read by experienced breast pathologists (HV, JLC) to assess if features on NLM required for diagnosing breast cancer were visible and to confirm that NLM images do not exhibit artifacts which confound diagnosis.

\section{RESULTS}

\section{Normal Human Breast Tissue}

Figure 2 shows (a) an NLM image and (b) a corresponding FFPE H\&E slide of normal breast tissue. Characteristic features of normal breast tissue were seen on both the NLM and FFPE H\&E images. Within the tissue, terminal duct lobular units (TDLUs) in the NLM images (Figure 2, green and brown) and the FFPE H\&E images (Figure 2, blue and orange) show clear correspondence. Individual acini (Figure 2, NLM: yellow; FFPE H\&E: pink) and larger nonterminal ducts (Figure 2, NLM: purple; FFPE H\&E: gray) are identifiable on NLM and match the FFPE H\&E image. Areas of normal adipocytes and collagen are both clear in NLM and FFPE H\&E (inset in green and blue and in Figures $2 \mathrm{a}$ and $\mathrm{b}$, respectively).

\section{Invasive Ductal Carcinoma}

Invasive ductal carcinoma (IDC) accounts for more than three quarters of all breast cancers. ${ }^{34}$ IDC is diagnosed when cancer cells break through the basement membrane of ducts and infiltrate the surrounding breast stroma. This pathology is characterized by malignant cells with variable prominent glandular structures infiltrating the breast parenchyma. Figure 3 shows (a) an NLM image with (b) a corresponding FFPE H\&E image of an IDC specimen. Areas of malignant cells can be seen in the NLM images (Figure 3, green) and FFPE H\&E images (Figure 3, blue) with areas of hyalinized stroma. Both images show infiltrating nests and glands of malignant cells, characteristic of IDC (Figure 3, NLM: yellow; FFPE H\&E: pink). There are inflamed vessels (Figure 3, NLM: brown; FFPE H\&E: orange) indicated by an increase in the endothelial cell size and an exudative infiltrate of lymphocytes. In the NLM image, like the FFPE H\&E image, typical

Figure 1 The method for processing and evaluating breast tissue using the rapid staining and imaging technology. Stain: freshly excised, unfixed tissue was stained in an acridine orange (AO), sulforhodamine 101 (SR101) solution and rinsed of excess stain using a 50\% ethanol/50\% water solution. Image: The light source, an ytterbium fiber laser, was scanned on to the sample through a $20 \times$ objective (OBJ) with resonant and non-resonant galvanometer actuated mirrors (scan unit). Fluorescent light from the specimen was separated from the two photon excitation light via a dichroic beam splitter (DBS) and further separated into components of fluorescent emission from AO and SR101 via a second DBS. Light was collected using a photomultiplier tube (PMT) for each channel. The PMT channels were rendered as virtual H\&E images on a graphics processing unit (GPU) and displayed in real time. (MR: Mirror). Evaluate according to standard-of-care: stained tissue was formalin-fixed and underwent standard paraffin-embedded histology processing using a commercial tissue processor (Tissue Tek VIP 5, Sakura, CA, USA), which includes ethanol dehydration, xylene rinsing, and paraffin infiltration. The standard processing extracted AO and SR101 to levels undetectable with NLM microscopy. Subsequent tissue evaluation can be performed in order to guide treatment and assess surgical outcome. 


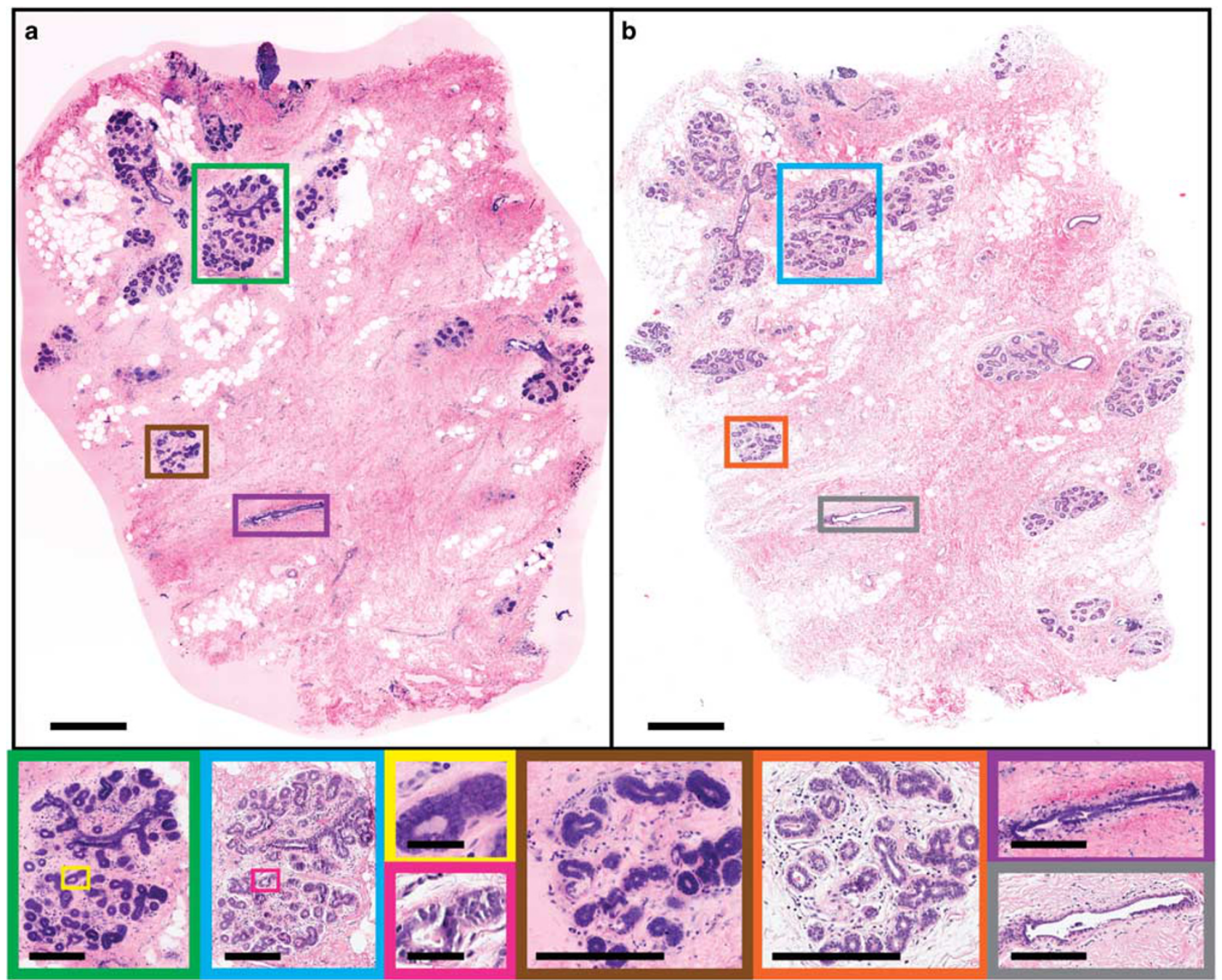

Figure 2 Normal human breast tissue. (a) NLM and (b) FFPE H\&E histology images (500 $\mu \mathrm{m}$ scale bar). Normal TDLUs (NLM: green and brown box; FFPE H\&E: blue and orange box) (250 $\mu \mathrm{m}$ scale bar), individual acini (NLM: yellow box; FFPE H\&E: pink box) (50 $\mu \mathrm{m}$ scale bar) and larger ducts (NLM: brown box; FFPE H\&E: gray box) (250 um scale bar) are shown magnified. NLM: https://slide-atlas.org/link/zmhbwg. FFPE H\&E: https://slide-atlas.org/link/noazj9.

malignant cell features, such as irregular shape and size of cells are evident.

\section{Invasive Lobular Carcinoma}

Invasive lobular carcinoma (ILC) is the second most common histological type of breast cancer. ${ }^{35}$ ILC begins in lobules of the breast and invades the surrounding stroma. Figure 4 shows an (a) NLM image and (b) a corresponding FFPE H\&E image of ILC. Classic ILC patterns of invasive lobular malignant cells infiltrating the surrounding breast stroma in a single file pattern are seen in the corresponding NLM (Figure 4, purple and brown) and the FFPE H\&E images (Figure 4, blue and orange). The single file strands of malignant cells are highlighted in the inset of Figure 4 outlined in yellow (NLM) and pink (FFPE H\&E). In addition, inflammatory infiltrates of lymphocytes, a typical response to a disease process such as cancer, along with a cross-sectional cut through a vessel, can be seen in NLM (Figure 4, green) and FFPE H\&E (Figure 4, gray).

\section{NLM at Multiple Depths For Serial Optical Sectioning}

NLM can generate images at different depths in tissue which are analogous to serial sectioned histology, decreasing the requirements on specimen flatness during imaging and enabling imaging through tissue surface artifacts due to electrocautery or debris from specimen handling. Figure 5 shows images acquired at multiple depths to $100 \mu \mathrm{m}$ below the tissue surface acquired with a constant laser power and detector gain. The detector gain was optimized for surface imaging. Digital gain in post processing was applied to the frames to match the average frame intensity throughout the stack of frames. This data shows that even with constant laser power and gain, it is possible to visualize tissue features $100 \mu \mathrm{m}$ below the tissue surface. 


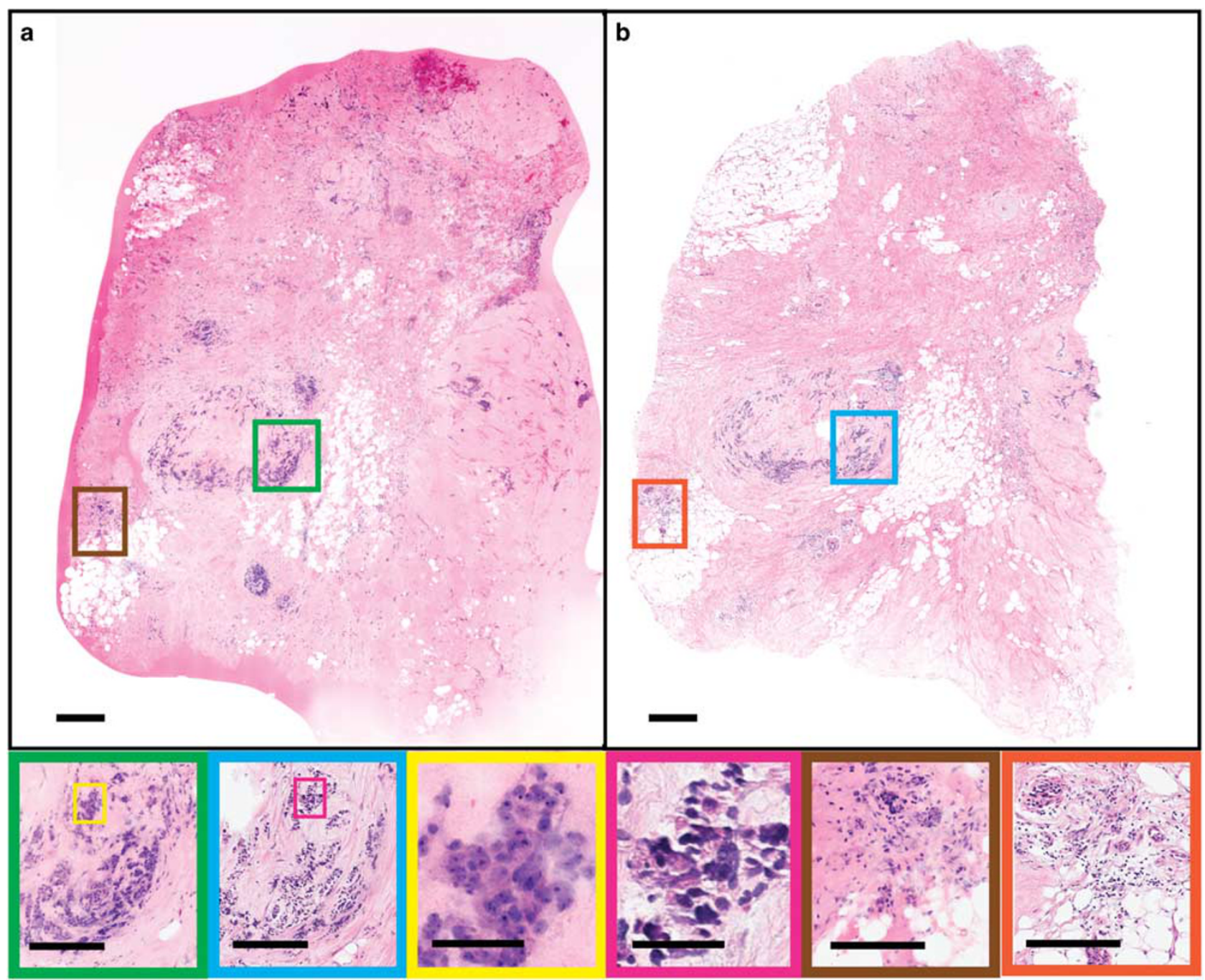

Figure 3 Human breast tissue showing invasive ductal carcinoma. (a) NLM and (b) FFPE H\&E images (500 $\mu$ m scale bar). Malignant cells are shown in the NLM (green) and FFPE H\&E images (blue) $(250 \mu \mathrm{m}$ scale bar) infiltrating the breast stroma, with a high power view shown in yellow (NLM) and pink (FFPE H\&E) (50 $\mu \mathrm{m}$ scale bar); inflamed vessels with a lymphocytic infiltrate are shown in brown (NLM) and orange (FFPE H\&E) (250 $\mu \mathrm{m}$ scale bar). NLM: https://slide-atlas.org/link/tfdr77. FFPE H\&E: https://slide-atlas.org/link/zatgkv.

\section{Extraction of AO and SR101 During Histological Processing}

Both SR101 and AO have high solubility in typical histology solvents such as ethanol and xylene and are expected to be extracted during processing. To assess the extraction of SR101 and $\mathrm{AO}$ as the result of routine histological processing, representative specimens of freshly excised, unfixed breast tissue were divided in half. The transected face of one half was stained with AO and SR101, while the matching face was unstained. The tissue was then fixed in formalin and processed using standard tissue processing on a vacuum infiltrating processor (VIP; Tissue Tek VIP 5, Sakura, CA, USA). The last tissue processing step is paraffin infiltration, however, paraffin is highly optically scattering, while infiltration produces geometric distortion of tissue. For purposes of comparison, the VIP was programmed to terminate prior to the paraffinization step (Supplementary Table S1). To provide images of unstained control tissue for comparison, second harmonic generation (SHG) contrast generated with an $800 \mathrm{~nm}$ Ti:S laser was used to image tissue without staining.

NLM images acquired with the same laser power and detector gain of the stained and unstained halves of the tissue are shown in Figure 6a prior to tissue processing (fluorescence: column 1, SHG: column 2) and after tissue processing (fluorescence: column 3, SHG: column 4). No fluorescence signal is detectable after tissue processing. The SHG images before and after tissue processing confirm that the images were acquired at roughly the same depth plane. Higher magnification regions of Figure 6a are shown in Figure $6 \mathrm{~b}$. Additionally, Figure $6 \mathrm{~b}$ shows images acquired with $20 \times$ higher detector gain. These images show a small amount of background autofluorescence, primarily from weak green fluorescent emission of collagen fibers. Within the background fluorescence, no nuclei are discernable. Enlarging a 


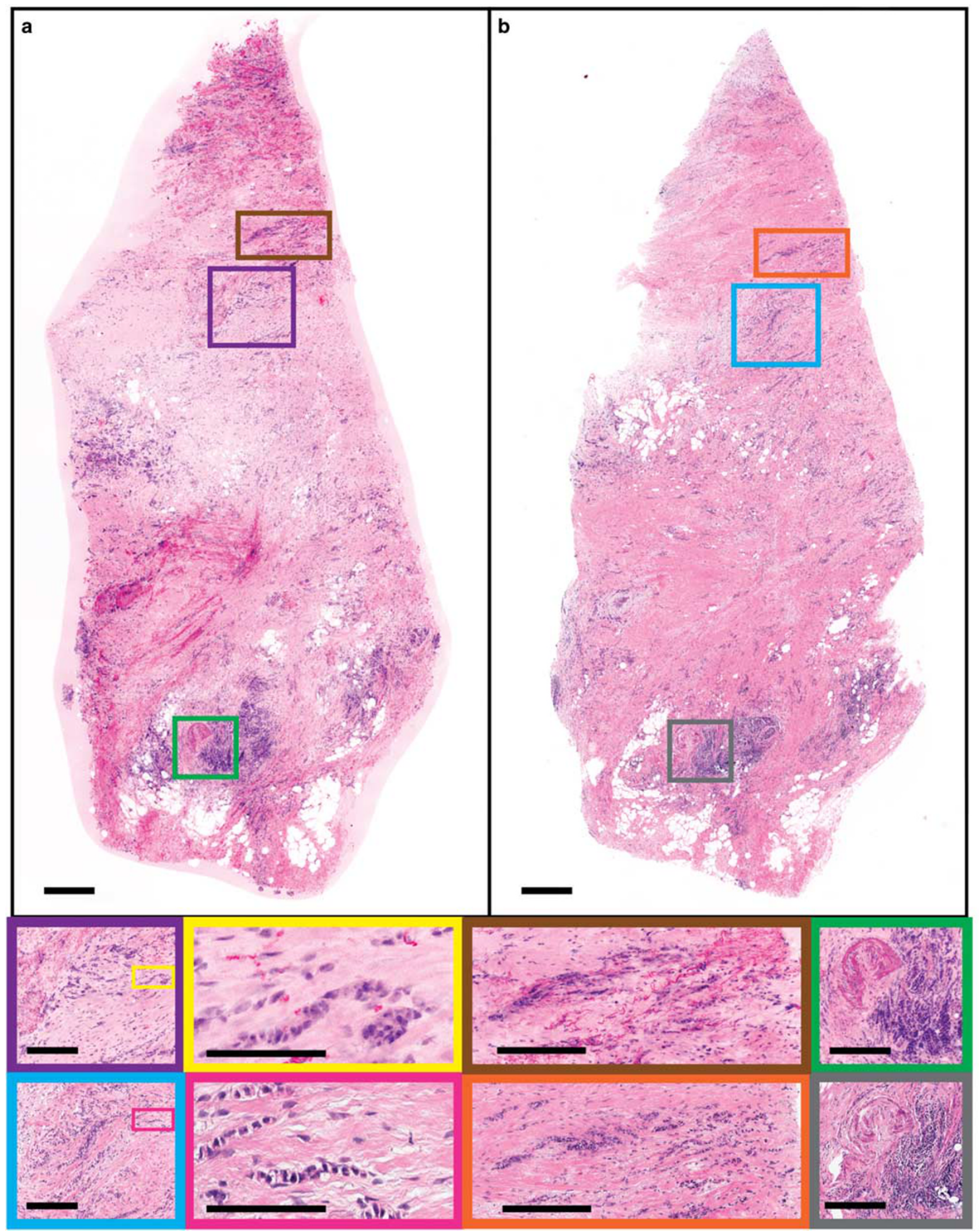

Figure 4 Human breast tissue showing invasive lobular carcinoma. (a) NLM image of freshly excised, unfixed human breast tissue with evident ILC and (b) corresponding FFPE H\&E histology ( $500 \mu \mathrm{m}$ scale bar). Many individual rows of malignant cells are in the NLM (purple and brown) and FFPE H\&E images (blue and orange) $(250 \mu \mathrm{m}$ scale bar). A high power view of the ILC single file pattern is shown in yellow (NLM) and pink (FFPE H\&E) (100 $\mu \mathrm{m}$ scale bar). A corresponding lymphocytic infiltration in the NLM and FFPE H\&E images is shown in green and gray, respectively (250 $\mu \mathrm{m}$ scale bar). NLM: https://slide-atlas.org/link/ghg3vq. FFPE H\&E: https://slide-atlas.org/link/h7vtjp. 


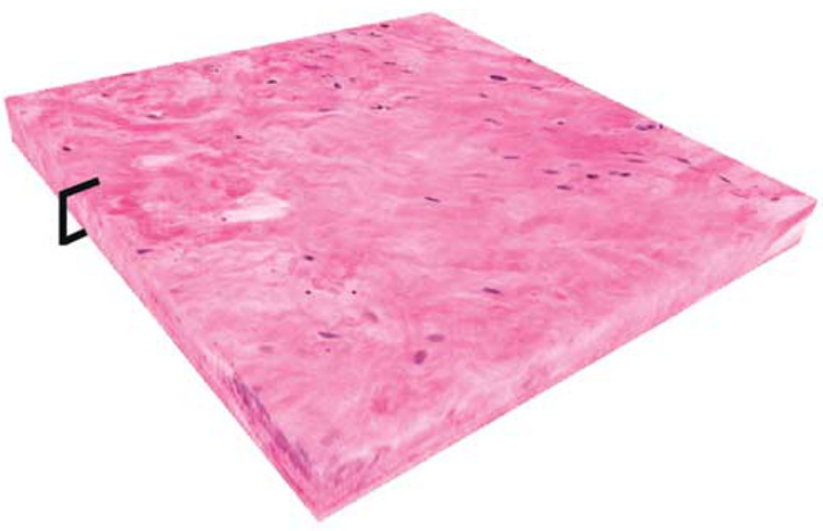

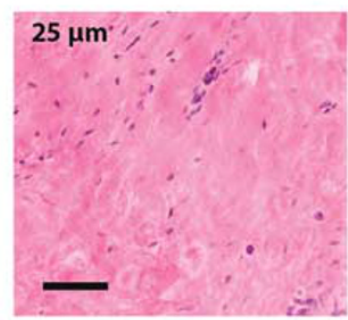

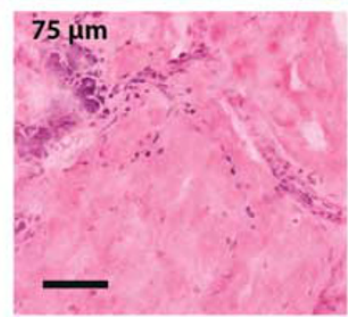

Figure 5 A $500 \times 500 \times 100 \mu \mathrm{m}$ three-dimensional stack of NLM images demonstrating imaging at different depths in normal breast tissue. The volumetric reconstruction shows image data from the surface to $100 \mu \mathrm{m}$ deep with digital gain applied to the images in post processing to match the average frame intensity without changing incident laser power or detector gain (scalebars: $100 \mu \mathrm{m}$ ). Selected NLM images from $25 \mu \mathrm{m}$, $50 \mu \mathrm{m}, 75 \mu \mathrm{m}$, and $100 \mu \mathrm{m}$ depths are shown.

region with nuclei shows a weak uniform signal due to autofluorescence leakage into the AO channel rather than the intensity spikes due to bound $\mathrm{AO}$ seen in the images acquired before processing (Figure 6c). Supplementary Figure S3 shows the fluorescence spectrum of xylene used to process $\mathrm{AO}$ stained tissue compared to a reference spectrum of AO taken before processing, indicating that xylene extracts $\mathrm{AO}$ from tissue without quenching $\mathrm{AO}$ fluorescence.

The reduction in fluorescent signal can be quantified by taking the ratio of the median normalized pixel intensities of the tissue before and after tissue processing for each data set and each channel (Supplementary Table S2). We found that the autofluorescence from the tissue is $\sim 1300$ times lower than the AO-stained nuclei in the AO channel and $\sim 12800$ times lower than the SR101 stained tissue in the SR101 channel. Because AO-stained nuclei are not present in the tissue after processing, we conclude that the intensity of $\mathrm{AO}$ staining is at least 1300 times less than before tissue processing and the intensity of SR101 staining is at least 12800 times less than before tissue processing. This reduction represents an upper bound on the maximum possible residual fluorescent signal that can be quantified given the autofluorescent background, however, since no residual nuclei can be observed even in regions with no apparent collagen, it is likely that the actual reduction is substantially higher.

\section{Verification of Noninterference With Fluorescence In Situ Hybridization}

To verify that extraction of AO and SR101 would enable HER2 FISH assays of fluorescently stained tissue, representative specimens of freshly excised, unfixed breast tissue were divided in half. One half was stained with the AO and SR101 protocol described earlier while the other was left unstained as a control. The tissue was then processed for standard FISH and examined by a trained pathologist (Supplementary Figure S4). The ratio of HER2:CEP 17 signals was calculated to be $\sim 1$ for both the treated and control tissue, which is negative for HER2 amplification. AO and SR101 fluorescence was not detectable in the specimens processed for FISH and there were no observable differences between the control and AO/SR101 stained tissue specimens.

\section{DISCUSSION}

NLM is attractive for intraoperative evaluation of pathology because it can optically section thick tissue, eliminating the labor-intensive and time-consuming embedding and physical sectioning step required for both paraffin sections and FSA, enabling real-time histological evaluation of excised tissue to guide surgery. NLM has been demonstrated to achieve high sensitivity and specificity for discrimination of normal and malignant breast tissue. ${ }^{21}$ Furthermore, the use of fluorescent contrast agents has several advantages, including rapid staining of live tissue, high NLM imaging speeds, high contrast, and, using the protocol described here, low fluorophore cost and compatibility with subsequent histological processing. However, the widespread use of fluorescence imaging and optical sectioning for histological evaluation of breast surgical pathology has been limited by substantial differences in the appearance of fluorescence images from standard FFPE H\&E images, lack of suitable fluorescent contrast agents, high cost and complexity of lasers, and concerns about interference with subsequent diagnostics following fluorescent staining. In this report, we have overcome these limitations and developed a protocol for NLM fluorescent nuclear staining with stromal counterstaining, combined with a VTM rendering algorithm ${ }^{33}$ to generate video rate virtual $\mathrm{H} \& \mathrm{E}$ images of freshly excised, unfixed tissue.

The study of representative breast surgical specimens presented here demonstrates a high degree of correspondence between FFPE H\&E and real-time NLM for normal breast tissue and common types of breast carcinoma. Review by 
a
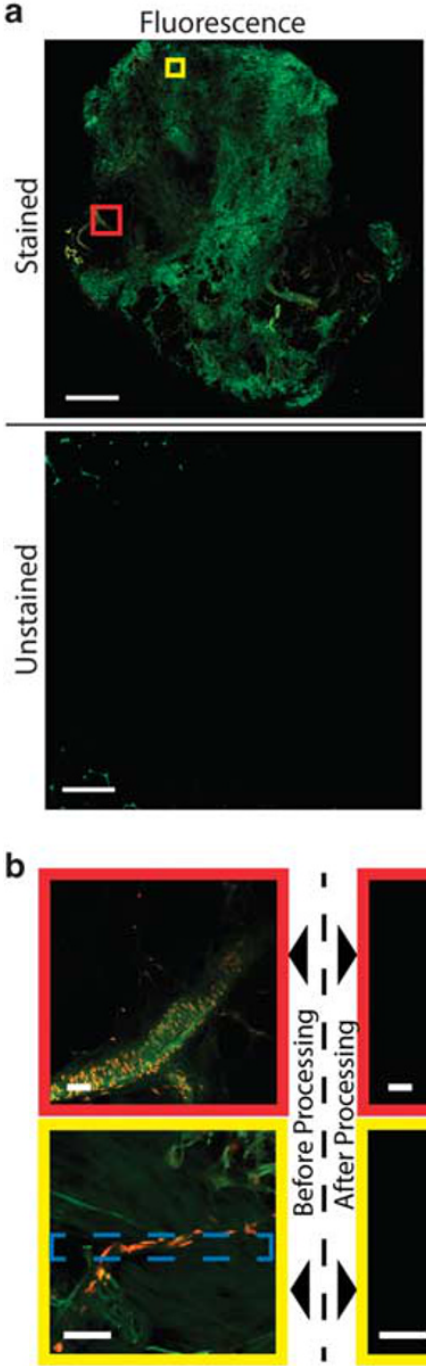
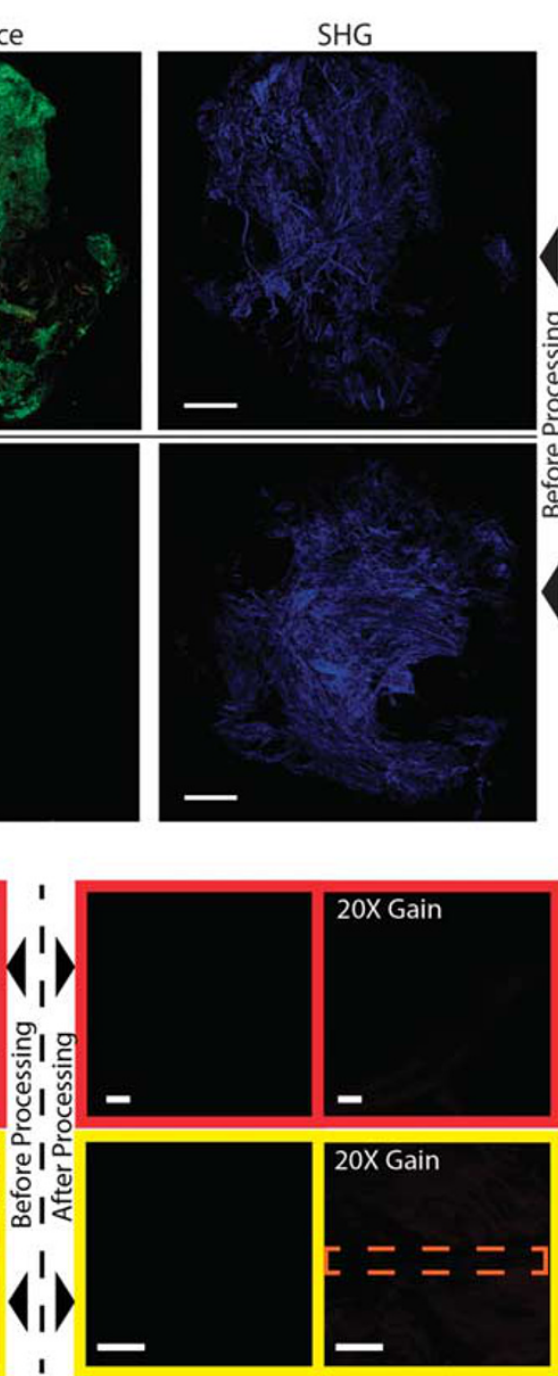

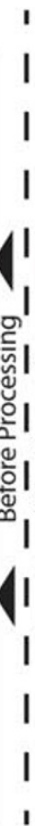

Fluorescence
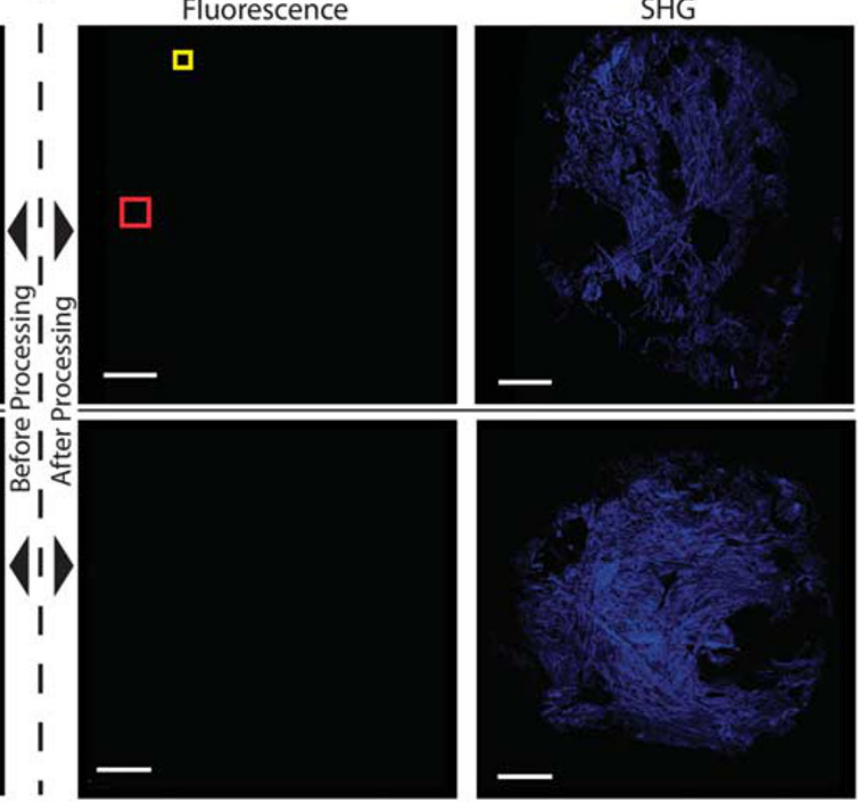

C

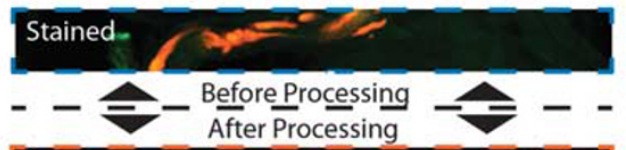

20X Gain

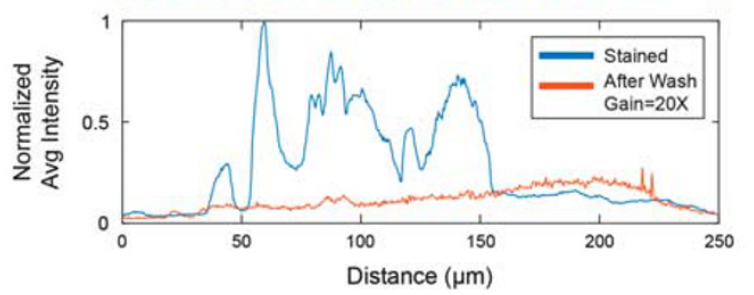

Figure 6 AO and SR101 are washed out to undetectable levels after standard tissue processing. (a) NLM fluorescent and SHG images before (first and second column) and after (third and fourth column) fixation and standard tissue processing of stained and unstained tissue. (Scale bars: $0.5 \mathrm{~mm}$ ). (b) High magnification fluorescent images of select areas of the specimens (a) before processing (left), after processing (center), and after processing with 20 times the detector gain (right) (scale bars: $50 \mu \mathrm{m}$ ) (c) A representative plot of the normalized average intensity of the AO channel from a frame in (b) before processing and after processing at $20 \times$ detector gain. The spikes in the 'stained' tissue correspond to fluorescence emitted from AO in nuclei. There are no corresponding spikes in the 'after wash' (after processing) graph and instead, there is a uniform background fluorescent signal present.

pathologists with expertise in breast cancer pathologies confirmed that diagnostically important features such as normal TDLUs (including benign individual acini and ducts) and normal fibrous and adipose stromal parenchyma, inflammation and biopsy site changes, invasive carcinoma with infiltrating nests, glands and, singly or single filing of malignant cells as well as in situ lobular and ductal carcinoma are present in NLM images associated with pathologies identified on standard FFPE H\&E histology. Despite minor differences between NLM and FFPE images such as geometric distortion and tissue shrinkage during fixation and physical sectioning, previous work has shown that NLM has nearequivalent sensitivity to conventional histology, indicating that minor differences in artifacts do not significantly impact interpretation of breast pathology. ${ }^{21}$ Further, quantitative image analysis of paraffin tissue sections indicates that both AO and SR101 have an extremely high degree of colocalization with hematoxylin and eosin, respectively (Supplementary Figure S5,Supplementary Table S3), indicating that our stains are a good approximation of conventional H\&E stains. Future clinical studies will be required to demonstrate improvements in surgical outcomes, however, the results presented here strongly suggest that NLM can provide diagnostic information equivalent to FFPE H\&E histology.

This manuscript demonstrates that stains enabling nearequivalent images to standard paraffin embedded histology can be rapidly applied to tissue and then extracted to undetectable levels by standard histological processing. Intraoperative assessment of breast tissue margins using the proposed protocol requires treating diagnostically relevant 
tissue with fluorescent stains. It is therefore important to minimize any impact on the tissue characteristics to prevent interference or reduced testing accuracy in subsequent immunohistochemical, FISH or other genetic tests that are necessary to maintain standards of patient care. A key advantage of the proposed protocol is that the fluorescent agents are selected to be highly soluble in conventional histology solvents. SR101, like eosin, is weakly bound to the tissue and highly soluble in methanol and is therefore extracted during formalin fixation. The less alcohol soluble $\mathrm{AO}$ is retained during fixation and dehydration, but extracted during xylene rinsing. The ability to apply stains and then extract them to undetectable levels using standard processing methods that are already validated for IHC and genetic assays is essential to incorporating advanced nonlinear imaging techniques into existing clinical workflows.

An advance towards clinical translation of fluorescence NLM presented in this manuscript is the replacement of complex, tunable Ti:S lasers with unamplified fixedwavelength fiber lasers by selecting contrast agents compatible with low power, $1 \mu \mathrm{m}$ excitation. While heroic efforts have been made to operate Ti:S lasers in the clinic, ${ }^{36}$ these systems are large and fragile, require regular maintenance, active cooling, vibration isolation, and substantial warm-up time, which precludes their use in routine clinical practice. In contrast, fiber lasers are compact and robust, do not require regular maintenance, cooling, or vibration isolation and power on rapidly. Consequently, system complexity, size, weight and cost are all greatly reduced. For example, the femtosecond fiber laser used here only occupies 12 " $\times 4$ " $\times 4$ " space and although the benchtop microscope used for this study occupies an additional 24 " $\times 30$ " $\times 20$ ", the fiber laser technology is small enough to enable NLM microscopes of comparable size to conventional light microscopes.

Another major advantage of NLM imaging at $1 \mu \mathrm{m}$ wavelength is the increased light penetration and imaging depth in tissue compared with NLM at shorter wavelengths or confocal microscopy (Supplementary Figure S2). Combined with rapid fluorescent stains with good tissue permeation, operation at $1 \mu \mathrm{m}$ enables visualization of tissue up to $100 \mu \mathrm{m}$ from the surface without adjusting incident power or detector gain, equivalent to 25 serial paraffin sections (Figure 5) without the processing delay and expense associated with taking sequential paraffin sections. The operator is able to view subsurface features in different regions of interest by panning and adjusting focal depth to evaluate the threedimensional structure of tissue in real time. In conventional breast histology, sequential serial paraffin sections are rarely taken due to the cost and time required to prepare and evaluate sequential slides. The ability to easily evaluate pathology in $3 \mathrm{D}$ is therefore unique to optical sectioning and may enable improved diagnostic sensitivity.

The ability to image below the surface of specimens may also be an important for intraoperative breast surgery applications because tissue surfaces often have contamination artifacts. Surgical tissue specimens typically have electrocautery, loose or displaced cells from excision or grossing, strongly fluorescent fibers from gauze, paper tissue or cutting boards, and other foreign material on the tissue surfaces. This may not be present on FFPE H\&E slides due to the tissue processing and microtoming which removes surface contaminants. Imaging modalities such as confocal microscopy have limited imaging range below tissue surfaces which may limit their diagnostic effectiveness in the presence of surface contamination. NLM can perform subsurface imaging to avoid surface contamination artifacts, which can be especially important for evaluating areas of dense, highly scattering cells such as in IDC.

In addition to the morbidity and the patient distress associated with incomplete resection and repeat surgeries, the cost of breast cancer treatment increases with each additional surgery, resulting in a substantial economic burden. ${ }^{37-39}$ Consequently, there is currently a shift towards a valuebased insurance payment method to incentivize increased quality of care, for example, reducing hospital readmissions, in order to control costs. ${ }^{40}$ The adoption of methods to assess margins intraoperatively promises to enable a reduction in the treatment cost while improving patient outcomes.

Supplementary Information accompanies the paper on the Laboratory Investigation website (http://www.laboratoryinvestigation.org)

\section{ACKNOWLEDGMENTS}

We thank Thorlabs for supplying microscope hardware and Kitware for providing SlideAtlas, an open-source platform for sharing our full-resolution FFPE H\&E slides and NLM images. This work was supported by the National Institutes of Health (CA178636-04, CA075289-19, CA183400-02) and Air Force Office of Scientific Research (FA9550-12-1-0551, FA9550-15-1-0473).

\section{DISCLOSURE/CONFLICT OF INTEREST}

The authors declare no conflict of interest.

1. American College Of Radiology. Practice guideline for the management of ductal carcinoma in-situ of the breast (DCIS). J Am Coll Surg 2007;205:145-161.

2. American College of Radiology. Practice guideline for the breast conservation therapy in the management of invasive breast carcinoma. J Am Coll Surg 2007;205:e2.

3. Sabel MS, Rogers K, Griffith K, et al. Residual disease after re-excision lumpectomy for close margins. J Surg Oncol 2009;99:99-103.

4. Kobbermann A, Unzeitig A, Xie X-J, et al. Impact of routine cavity shave margins on breast cancer re-excision rates. Ann Surg Oncol 2011;18: 1349-1355.

5. Morrow M, Jagsi R, Alderman AK, et al. Surgeon recommendations and receipt of mastectomy for treatment of breast cancer. JAMA 2009;302: 1551-1556.

6. McCahill LE, Single RM, Aiello Bowles EJ, et al. Variability in reexcision following breast conservation surgery. JAMA 2012;307:467-475.

7. Fleming FJ, Hill ADK, Mc Dermott EW, et al. Intraoperative margin assessment and re-excision rate in breast conserving surgery. Eur J Surg Oncol 2004;30:233-237.

8. Huston TL, Pigalarga R, Osborne MP, et al. The influence of additional surgical margins on the total specimen volume excised and the reoperative rate after breast-conserving surgery. Am J Surg 2006;192: 509-512. 
9. Ferreiro J, Gisvold J, Bostwick D. Accuracy of frozen-section diagnosis of mammographically directed breast biopsies. Results of 1,490 consecutive cases. Am J Surg Pathol 1995;19:1267-1271.

10. Menes TS, Tartter PI, Mizrachi $\mathrm{H}$, et al. Touch preparation or frozen section for intraoperative detection of sentinel lymph node metastases from breast cancer. Ann Surg Oncol 2003;10:1166-1170.

11. Allen TC. The incredible shrinking billing codes. Arch Pathol Lab Med 2014;138:593-594.

12. Boppart SA, Richards-Kortum R. Point-of-care and point-of-procedure optical imaging technologies for primary care and global health. Sci Transl Med 2014;6:253rv2-253rv2.

13. Leigh SY, Liu JTC. Multi-color miniature dual-axis confocal microscope for point-of-care pathology. Opt Lett 2012;37:2430-2432.

14. Bini J, Spain J, Nehal K, et al. Confocal mosaicing microscopy of human skin ex vivo: spectral analysis for digital staining to simulate histologylike appearance. J Biomed Opt 2011;16:76008.

15. Chung VQ, Dwyer PJ, Nehal KS, et al. Use of ex vivo confocal scanning laser microscopy during Mohs surgery for nonmelanoma skin cancers. Dermatologic Surg 2004;30:1470-1478.

16. Gareau DS, Karen JK, Dusza SW, et al. Sensitivity and specificity for detecting basal cell carcinomas in Mohs excisions with confocal fluorescence mosaicing microscopy. J Biomed Opt 2009;14: 34012-34017.

17. Yoshitake T, Giacomelli MG, Cahill LC, et al. Direct comparison between confocal and multiphoton microscopy for rapid histopathological evaluation of unfixed human breast tissue. J Biomed Opt 2016;21: 126021.

18. $\mathrm{Fu} \mathrm{HL}$, Mueller $\mathrm{J}$, Javid $\mathrm{MP}$, et al. Optimization of a widefield structured illumination microscope for non-destructive assessment and quantification of nuclear features in tumor margins of a primary mouse model of sarcoma. PLoS One 2013;8:e68868.

19. Schlichenmeyer TC, Wang M, Elfer $\mathrm{KN}$, et al. Video-rate structured illumination microscopy for high-throughput imaging of large tissue areas. Biomed Opt Express 2014;5:366-377.

20. Orringer DA, Pandian B, Niknafs YS, et al. Rapid intraoperative histology of unprocessed surgical specimens via fibre-laser-based stimulated Raman scattering microscopy. Nat Biomed Eng 2017;1:27.

21. Tao YK, Shen D, Sheikine $Y$, et al. Assessment of breast pathologies using nonlinear microscopy. Proc Natl Acad Sci USA 2014;111: 15304-15309.

22. Tu H, Liu Y, Turchinovich D, et al. Stain-free histopathology by programmable supercontinuum pulses. Nat Photonics 2016;10:534-540.

23. Chen SY, Chen SU, Wu HY, et al. In vivo virtual biopsy of human skin by using noninvasive higher harmonic generation microscopy. IEEE J Sel Top Quantum Electron 2010;16:478-492.

24. Tang S, Liu J, Krasieva TB, et al. Developing compact multiphoton systems using femtosecond fiber lasers. J Biomed Opt 2009;14:30508.
25. Millard AC, Wiseman PW, Fittinghoff DN, et al. Third-harmonic generation microscopy by use of a compact, femtosecond fiber laser source. Appl Opt 1999;38:7393.

26. Jacques SL. Optical properties of biological tissues: a review. Phys Med Biol 2013;58:5007-5008.

27. Balu M, Saytashev I, Hou J, et al. Sub-40 fs, 1060-nm Yb-fiber laser enhances penetration depth in nonlinear optical microscopy of human skin. J Biomed Opt 2015;20:120501.

28. Kobat D, Durst ME, Nishimura $N$, et al. Deep tissue multiphoton microscopy using longer wavelength excitation. Opt Express 2009;17: 13354.

29. Olson E, Levene MJ, Torres R. Multiphoton microscopy with clearing for three dimensional histology of kidney biopsies. Biomed Opt Express 2016;7:3089-3096.

30. Ragazzi M, Piana S, Longo C, et al. Fluorescence confocal microscopy for pathologists. Mod Pathol 2013;27:1-12.

31. Gareau D, Bar A, Snaveley N, et al. Tri-modal confocal mosaics detect residual invasive squamous cell carcinoma in Mohs surgical excisions. J Biomed Opt 2012;17:66018.

32. Robertson T, Bunel F, Roberts M. Fluorescein Derivatives in Intravital Fluorescence Imaging. Cells 2013;2:591-606.

33. Giacomelli MG, Husvogt $\mathrm{L}$, Vardeh $\mathrm{H}$, et al. Virtual hematoxylin and eosin transillumination microscopy using epi-fluorescence imaging. PLOS ONE 2016;11:e0159337.

34. Li Cl, Uribe DJ, Daling JR. Clinical characteristics of different histologic types of breast cancer. Br J Cancer 2005;93:1046-1052.

35. $\mathrm{Li} \mathrm{Cl}$, Malone $\mathrm{KE}$, Porter $\mathrm{PL}$, et al. Relationship between long durations and different regimens of hormone therapy and risk of breast cancer. JAMA 2003;289:3254-3263.

36. Giacomelli MG, Yoshitake $T$, Husvogt $L$, et al. Design of a portable wide field of view GPU-accelerated multiphoton imaging system for realtime imaging of breast surgical specimens. In: Periasamy A, So PTC, König K. Proc SPIE 9712, Multiphoton Microscopy in the Biomedical Sciences XVI., 2016: 97121G.

37. Zheng $Z$, Yabroff KR, Guy GP, et al. Annual medical expenditure and productivity loss among colorectal, female breast, and prostate cancer survivors in the United States. J Natl Cancer Inst 2016;108: djv382.

38. Metcalfe LN, Zysk AM, Edelman G, et al. Beyond the margins: Economic costs and complication rates associated with repeated breastconserving surgeries. J Clin Oncol, 34 2016; (suppl):1050.

39. Uecker JM, Bui EH, Foulkrod $\mathrm{KH}$, et al. Intraoperative assessment of breast cancer specimens decreases cost and number of reoperations. Am Surg 2011;77:342-344.

40. Chien AT, Rosenthal MB. Medicare's physician value-based payment modifier-will the tectonic shift create waves? N Engl J Med 2013;369: 2076-2078. 